Envelhecer com qualidade e participação: experiência de um curso de residência multiprofissional em saúde

\title{
Aging with quality and participation: experience in a multiprofessional health residency course
}

\section{Envejecer con calidad y participación: experiencia de un curso de residencia multiprofessional en salud}

Recebido: 22/11/2016

Aprovado: 14/05/2017

Publicado: 03/08/2017
Priscila Maitara Avelino Ribeiro1

Mariana Campos de Sousa ${ }^{2}$

Marta Regina Farinelli ${ }^{3}$

Este é um relato de experiência que tem como objetivo descrever o trabalho socioeducativo desenvolvido com pessoas idosas do grupo HiperDia de uma Unidade Básica de Saúde (UBS). A atividade foi realizada no período de março de 2016 a março de 2017. Atualmente, estão inscritos 578 usuários na idade de 60 a 80 anos. Foi utilizada no grupo educativo a metodologia dialógica, por meio de atividades pautadas na promoção da saúde e no envelhecimento ativo e saudável. Como facilitadores, foram envolvidos residentes multiprofissionais e outros profissionais da unidade de saúde. Constatou-se a melhoria na autonomia, independência e participação dos (as) idosos (as) no seu processo de saúde e cuidado, com influência em sua qualidade de vida.

Descritores: Envelhecimento; Idoso; Qualidade de vida.

This is an experience report whose objective is to describe the social-educational work developed with elders from the group HiperDia in a Primary Health Care Unit (UBS). The activity was conducted from March 2016 to May 2017 Currently, 578 users from 60 to 80 years of age are registered. A dialogic methodology was was used in the group, through activities based on health promotion and active healthy aging. Multiprofessional residents and other professionals from the health unit were the facilitators and conducted the group. It was noted an improvement in the autonomy, independence and participation of the elders in their process of health and care, influencing in their quality of life.

Descriptors: Aging; Aged; Quality of life.

Este es un relato de experiencia que tiene como objetivo describir el trabajo socioeducativo desarrollado con personas ancianas del grupo HiperDia de una Unidad Básica de Salud (UBS). La actividad fue realizada en el período de marzo de 2016 a marzo de 2017. Actualmente, están inscriptos 578 usuarios con edad de 60 a 80 años. Fue utilizada en el grupo educativo la metodología dialógica, por medio de actividades pautadas en la promoción de la salud, envejecimiento activo y saludable y envolvió como facilitadores residentes multiprofesionales y otros profesionales de la unidad de salud. Se constató la mejoría en la autonomía, independencia y participación de los ancianos en su proceso de salud y cuidado, con influencia en la calidad de vida de estos.

Descriptores: Envejecimiento; Anciano; Calidad de vida.

\footnotetext{
${ }^{1}$ Assistente Social. Especialista em Políticas Públicas, Sociais e de Saúde. Especializanda em Saúde do Adulto na modalidade da Residência Interdisciplinar e Multiprofissional em Saúde/Universidade Federal do Triângulo Mineiro - UFTM, Uberaba/MG/Brasil. ORCID 0000-0001-50454949 E-mail: maitara@hotmail.com

2 Enfermeira. Especialista em Saúde do Idoso na modalidade Residência Integrada e Multiprofissional em Saúde. Mestranda em Atenção à Saúde pelo Programa de Pós Graduação em Atenção à Saúde da UFTM, Uberaba/MG/Brasil. ORCID 0000-0001-6803212X. E-mail: mariana_camposdesousa@hotmail.com

${ }^{3}$ Assistente Social. Bacharel em Direito. Mestre e Doutora em Serviço Social. Professora Adjunta IV do Curso de Serviço Social, do Programa de Pós Graduação em Psicologia e, da Residência Integrada e Multiprofissional em Saúde da Universidade Federal do Triângulo Mineiro (RIMS/UFTM), Uberaba/MG/Brasil. ORCID 0000-0003-0536-4017 E-mail: martafarinelli@gmail.com
} 


\section{INTRODUÇÃO}

$\mathrm{N}$ os últimos anos o debate acerca do processo de envelhecimento ativo tem sido tema de vários pesquisadores e estudiosos, em virtude da longevidade da população mundial ${ }^{1}$. Assim, a busca pela qualidade de vida das pessoas idosas acaba se tornando um desafio para o poder público, para os profissionais que atuam com este segmento populacional, bem como para toda a sociedade que se relaciona com os (as) idosos (as), quer seja no espaço de trabalho ou no convívio familiar.

No Brasil, a Constituição Federal de 1988 define a proteção social, configurando-a como um sistema de Seguridade Social que compreende as políticas da previdência social, da assistência social e da saúde, sendo as mesmas reconhecidas como políticas públicas e direitos sociais destinadas a todos os segmentos populacionais.

No âmbito da Política de Saúde, dentre os documentos legais que garantem o direito à saúde, destaca-se o Sistema Único de Saúde (SUS) que surgiu há mais de 25 anos como uma nova configuração política e organizacional para o rearranjo dos serviços e ações de saúde. Seu objetivo é garantir uma assistência à saúde em todo país, sem distinções e discriminações. O SUS possui princípios doutrinários para a sua efetivação, que consistem em: Universalidade, Integralidade e Equidade. Além disso, as diretrizes que regem a sua organização são: Regionalização e Hierarquização, Resolubilidade, Descentralização e Participação dos Cidadãos. Tais princípios e diretrizes norteiam os serviços de saúde no país ${ }^{2}$.

Por sua vez, a Atenção Básica é umas das propostas do Ministério da Saúde para implementar os princípios do SUS nos serviços de saúde. Caracteriza-se por um conjunto de ações de saúde, no âmbito individual e coletivo, que envolvem a promoção e a proteção da saúde, a prevenção de agravos, o diagnóstico, o tratamento, a reabilitação, a redução de danos e a manutenção da saúde, com o objetivo de oferecer uma assistência integral que melhore a situação de saúde e desenvolva a autonomia das pessoas. Ela deve ser o primeiro contato dos usuários com o serviço de saúde, a principal porta de entrada e o centro de comunicação com toda a Rede de Atenção à Saúde ${ }^{3}$.

A Atenção Básica engloba, dentre outras iniciativas, a Estratégia de Saúde da Família (ESF). Esta surge na década de 1990, inicialmente configurada como Programa de Saúde da Família, como uma ousada proposta para a reestruturação do sistema de saúde, organizando a atenção primária e substituindo os modelos tradicionais existentes ${ }^{3}$. A ESF está centrada na família, que é assistida em seu ambiente físico e social, o que possibilita às equipes da Saúde da Família uma compreensão do processo saúdedoença da população atendida, permitindo uma melhora na qualidade da assistência e, consequentemente, na qualidade de vida dos usuários.

Os atendimentos e cuidados oferecidos na Atenção Primária são complexos, ao contrário do que muitos pensam, e precisam dar conta das necessidades de saúde da população, em nível individual e/ou coletivo, de forma que as ações interfiram na saúde e na autonomia das pessoas e nos determinantes e condicionantes de saúde da comunidade ${ }^{4}$.

Neste contexto, o Programa de Hipertensão Arterial e Diabetes (HiperDia) propõe o acompanhamento de pessoas/usuárias hipertensas e/ou diabéticas, tendo como um dos objetivos vincular a pessoa à Unidade Básica de Saúde (UBS) e à Equipe de Saúde da Família (ESF) de referência, realizar uma assistência contínua e de qualidade.

O HiperDia é consolidado por intermédio dos profissionais atuantes na Estratégia Saúde da Família (ESF), que implementam as ações aos usuários e demonstram a importância do cuidado com a Hipertensão Arterial Sistêmica (HAS) e o Diabetes Mellitus (DM). Tais profissionais trabalham com modelos de atenção mais responsáveis, com vista a estimular nos usuários o autocuidado, coresponsabilizando-os pela sua própria saúde ${ }^{5}$.

Nesta perspectiva, o grupo de Residentes Multiprofissionais das seguintes 
categorias: Assistente Social, Enfermeira, Fisioterapeuta, profissional de Educação Física, da área de concentração da saúde do idoso do Programa de Residência Integrada Multiprofissional em Saúde da Universidade Federal do Triângulo Mineiro (UFTM), juntamente com a equipe de Estratégia de Saúde da Família (ESF) em uma UBS do município de Uberaba-MG, desenvolvem atividades com o Grupo HiperDia.

A Pesquisa Nacional de Saúde (2013), realizada pelo Ministério da Saúde em parceria com o IBGE, mostra que o diabetes atinge 9,1 milhões de brasileiros - o que corresponde a 6,2\% da população de 18 anos ou mais de idade. As mulheres (7\%) apresentaram maior proporção da doença do que os homens $(5,4 \%)$. Os percentuais de prevalência da doença por faixa etária são: $0,6 \%$ entre 18 a 29 anos; $5 \%$ de 30 a 59 anos; $14,5 \%$ entre 60 e 64 anos e $19,9 \%$ entre 65 e 74 anos. Para aqueles que tinham 75 anos ou mais de idade, o percentual foi de 19,6\%. Já a proporção de indivíduos de 18 anos ou mais que referem diagnóstico de hipertensão arterial no Brasil foi de 21,4\% em 2013, o que corresponde a 31,3 milhões de pessoas ${ }^{6}$.

As mulheres $(24,2 \%)$ apresentavam maior proporção da doença que os homens (18,3\%). 0 percentual de prevalência da doença aumentava com a idade: 2,8\% para pessoas de 18 a 29 anos; 20,6\% de 30 a 59 anos; $44,4 \%$ de 60 e 64 anos, $52,7 \%$ de 65 a 74 anos de idade e $55 \%$ entre as pessoas de 75 anos ou mais de idade 6 .

Com o processo de envelhecimento físico e biológico, e sem fatores ativos saudáveis, um número maior de doenças crônicas pode ocorrer e se desenvolver, havendo, portanto um maior número de pessoas diabéticas. Assim, é importante pensar como a sociedade está envelhecendo na atualidade, além de se trabalhar a importância do envelhecimento ativo saudável durante todo o ciclo de vida e não somente com o aumento da idade.

Desta forma, partindo da premissa que toda pessoa necessita pensar o processo do envelhecimento em sua totalidade, o presente relato tem como objetivo compartilhar o trabalho socioeducativo desenvolvido com pessoas idosas do Grupo HiperDia de uma Unidade Básica de Saúde (UBS) -de Uberaba MG, tendo como foco a relevância do processo de envelhecimento humano ativo e saudável.

Para fins deste relato de experiência, se considerará envelhecimento ativo de acordo com a Organização Mundial de Saúde ${ }^{7}$ como:

"o processo de otimização das oportunidades de saúde, participação e segurança, com o objetivo de melhorar a qualidade de vida à medida que as pessoas ficam mais velhas". "O envelhecimento ativo aplica-se tanto a indivíduos quanto a grupos populacionais".

Entende-se que o envelhecimento traz transformações biológicas, podendo acarretar doenças crônicas, metabolismo lento, aparência desgastada, menor destreza e mobilidade. Ainda assim, estes fatores podem ser retardados ou ter seu impacto diminuído, se o processo de envelhecimento for com qualidade de vida.

Com vistas a desenvolver ações com enfoque no envelhecimento ativo e saudável propôs-se a realização de um projeto de extensão pautado numa proposta de educação em saúde.

A Educação em saúde compreendida como fator de promoção e proteção à saúde e a estratégia para a conquista dos direitos de cidadania. A Educação em saúde de acordo com o Ministério da Saúde ${ }^{8}$ é:

"a tradução da proposta constitucional em prática requer o desenvolvimento da consciência sanitária da população e dos governantes para que o direito à saúde seja encarado como prioridade".

Assim, objetivo deste artigo é descrever o trabalho socioeducativo desenvolvido com pessoas idosas do grupo HiperDia de uma Unidade Básica de Saúde (UBS) de Uberaba(MG).

\section{MÉTODO}

0 estudo caracteriza-se como um relato de experiência, de um projeto de extensão realizado por meio do trabalho socioeducativo pelos residentes do Programa de Residência Integrada e Multiprofissional da Universidade Federal do Triângulo Mineiro - UFTM e equipe de Estratégia de Saúde da Família (ESF) em uma UBS do município de Uberaba-MG.

0 referido projeto socioeducativo se realiza desde 2011, para efeitos deste relato, 
se descreverá a atividade de 2016 a 2017.

A atividade utilizou perspectivas dialógicas e contou com uma equipe multiprofissional que faz parte da RIMS concentração saúde do idoso.

\section{RESULTADOS}

Estes grupos são desenvolvidos desde 2011 pela equipe de saúde da UBS (composta por enfermeiros, agentes comunitários de saúde, médicos, dentistas, técnicos em enfermagem), sendo que houve contribuição neste mesmo ano da primeira turma de residentes do referido Programa de Residência Integrado e Multiprofissional em Saúde.

A facilidade em desenvolver as atividades se deu pelo fato de que o quadro de profissionais/residentes inclui diversas categorias profissionais, o que amplia as discussões sob vários olhares e propicia aos participantes conhecimentos diferenciados.

A implantação deste projeto de extensão e prestação de serviços em 2016 foi realizada em três etapas. Inicialmente realizou-se a revisão bibliográfica de assuntos relativos à saúde, promoção da saúde, envelhecimento ativo e saudável, direitos sociais, hipertensão, diabetes, entre outros que contribuíram para o fortalecimento teórico dos facilitadores do grupo HiperDia. Também foi realizada uma pesquisa e coleta de informações em documentos legais das políticas de atendimento à pessoa idosa.

Os grupos de participantes do HiperDia se reúnem uma vez por semana (terças, quartas e quintas) e em cada dia atende-se às demandas de um determinado bairro, com área de abrangência da UBS.

No grupo é realizado o acolhimento dos participantes (em sua grande maioria pessoas idosas) com diagnóstico de diabetes e hipertensão arterial, tendo a presença controlada por "cartões" que contêm os dados dos participantes e outras informações pertinentes à hipertensão e diabetes.

Em um segundo momento é realizado uma atividade com um tema norteador como: diabetes, alimentação, quedas, osteoporoses, hanseníase, pé-diabético, direitos sociais, relevância de se fazer exercícios físicos diariamente, feridas, envelhecimento ativo, entre outros assuntos que os participantes demandam ou sugerem para discussão.

Em cada encontro/dia um profissional da equipe de saúde da UBS e /ou equipe de residentes apresenta, discute e esclarece um tema consoante à sua área de formação profissional. Neste momento são realizadas dinâmicas em grupo, explanação dialogada, músicas, imagens, folhetos autoexplicativos, entre outros recursos que contribuem para facilitar o diálogo, a participação e o interesse dos presentes.

Em um terceiro e último momento do dia inicia-se a "pesagem", medição da circunferência abdominal, aferição da pressão arterial, teste de glicemia capilar, além de esclarecimentos de dúvidas acerca de alimentação, controle da diabetes, uso de medicamentos, entre outros que possam surgir. Posteriormente, e se necessário for, a pessoa é encaminhada para que possa consultar com o médico generalista da UBS.

As atividades ocorreram de março de 2016 até março de 2017 e visam: disseminar temas de educação em saúde que possam contribuir com a participação e a autonomia dos (as) idosos (as) para com sua saúde; buscar o atendimento para controle de hipertensão e diabetes; orientar os participantes nas dúvidas referentes a todo o processo de sua saúde; propor um grupo que contribua para o envelhecimento ativo e cuidado com a saúde.

A equipe de facilitadores é composta por: 2 enfermeiros da ESF; cerca de 15 agentes comunitários de saúde; 2 técnicos de enfermagem; 1 discente de graduação em Nutrição; 3 discentes de graduação em Enfermagem; 2 Residentes/Enfermeiras; 2 Residentes/Fisioterapeutas; 2 Residentes/ profissionais de Educação Física e 1 Residente/Assistente Social.

As atividades são realizadas em sistema de revezamento: ora equipe UBS, ora equipe de residentes e após cada atividade os facilitadores registram e atualizam os dados dos participantes na ficha do SISVAN-Sistema de Vigilância Alimentar Nutricional e na ficha de acompanhamento de Hipertensos e Diabéticos.

De forma contínua as equipes realizam 
avaliação para garantir o atendimento integrado e prevenir os agravos causados pela elevação de pressão arterial ou altos níveis de glicemia capilar.

A equipe da ESF da UBS busca periodicamente aperfeiçoamento através de cursos de capacitação e formação continuada por meio da Secretaria Estadual de Saúde, e por professores da (Universidade Federal do Triângulo Mineiro (UFTM), para qualificar o atendimento proporcionado ao grupo do HiperDia, e contribuir para a intervenção realizada junto aos usuários.

\section{DISCUSSÃO}

A execução do projeto ocorreu por meio de abordagem dialógica ${ }^{9}$ e articulada em dois eixos norteadores: um relativo à aquisição do conhecimento e das habilidades de aprender e fazer, e o outro relacionado à convivência e ao autoconhecimento, tendo em vista a promoção da saúde, o controle de doenças crônicas, o incentivo à autonomia e à independência de forma participativa $\mathrm{e}$ coletiva, na busca de envelhecimento ativo e melhoria na qualidade de vida.

As atividades realizadas no grupo HiperDia estão fundamentadas na Política Nacional de Promoção da Saúde, pois atendem aos critérios de participação e do cuidado às pessoas com doença crônica, além de fomentar a participação e a autonomia da pessoa nos seus cuidados de saúde. Tais ações são pautadas no envelhecimento ativo, pois buscam no cuidado da pessoa idosa e no processo de envelhecimento participação e compreensão dos fatores que influenciam em um envelhecimento ativo, com qualidade de vida, autonomia e independência ${ }^{10}$.

Uma questão a se considerar no trabalho com a população idosa é o processo de envelhecimento, que é inerente à pessoa humana além de se caracterizar como um processo universal, progressivo e gradual da pessoa, de envolver fatores de ordem genética, biológica, sociopolítica, ambiental, psicológica e cultural ${ }^{11}$.

As ações do projeto de intervenção têm se intensificado e verifica-se que a metodologia dialógica proporcionou a melhora e a adesão dos usuários ao tratamento anti-hipertensivo e/ou antidiabético na APS, bem como sua integração com a comunidade. Constatou-se também, pelo acompanhamento realizado pelas equipes, que o comparecimento dos usuários na UBS tem aumentado de forma considerável.

O Sistema de Cadastramento e Acompanhamento de Hipertensos e Diabéticos (HiperDia) é um sistema informatizado nacional de cadastro e acompanhamento de portadores de diabetes e hipertensão nas unidades básicas de saúde ${ }^{12}$. Esse sistema permite o monitoramento e o gerenciamento de informações para a aquisição, dispensação e distribuição de medicamentos de forma regular e organizada 5 .

Além do cadastro e da distribuição de medicamentos, o sistema permite o acompanhamento e a definição do perfil epidemiológico desta população, e a organização de grupos de educação em saúde que poderão modificar os hábitos de vida errados dos usuários, melhorando a qualidade de vida dessas pessoas e reduzindo o custo social ${ }^{13}$.

A proposta da RIMS tem provocado o dialogo acerca da necessidade e relevância do cuidado nas práticas de saúde, por meio de ações de promoção da saúde, prevenção de doenças, recuperação e reabilitação, ratificando a contribuição do trabalho interdisciplinar e multiprofissional, incluindo o reconhecimento da contribuição de outros saberes na assistência à saúde, possibilitando a compreensão dos sujeitos na sua totalidade.

No decorrer dos encontros, as equipes de facilitadores compostas por profissionais de saúde da UBS e profissionais residentes foram intensificando as discussões, e introduzindo ações com vistas ao envelhecimento ativo da população atendida no HiperDia.

As atividades realizadas no grupo de HiperDia buscam promoção da saúde e prevenção de doenças e agravos que possam prejudicar a condição de saúde da população atendida na UBS. O grupo trabalha diretamente com a população idosa, buscando contribuir com o processo de envelhecimento 
ativo por meio de intervenções diretas e através da educação em saúde.

As atividades desenvolvidas no grupo de HiperDia estão em consonância com o conceito de promoção da saúde, apontado como:

"processo de capacitação da comunidade para atuar na melhoria da sua qualidade de vida e saúde, incluindo maior participação no controle desse processo" 14 .

Isto, pois, busca de alguma forma capacitar a pessoa idosa para o controle e melhoria da sua saúde.

Este processo ainda permite que as pessoas desenvolvam seu potencial para o bem-estar físico, social e mental ao longo do curso da vida, e que essas pessoas:

"participem da sociedade de acordo com suas necessidades, desejos e capacidades; ao mesmo tempo, propicia proteção, segurança e cuidados adequados, quando necessários"7.

É nesta perspectiva que o grupo HiperDia nesta unidade de saúde e com esses atores - profissionais da RIMS - tem trabalhado, buscando efetivar uma intervenção pautada no envelhecimento ativo e saudável, e não somente no envelhecimento físico e aparente, fazendo com que as pessoas idosas possam compreender melhor seu processo de saúde e cuidado.

\section{CONCLUSÃO}

É imperioso que o debate sobre a participação e os cuidados acerca da saúde seja ampliado e disseminado pela equipe multiprofissional para a população idosa.

A participação, a autonomia nos assuntos referentes à saúde, bem como as demais dimensões da vida humana, buscam a qualidade de vida e um processo de envelhecimento ativo e saudável.

Neste âmbito pode-se afirmar que a realização das atividades de educação em saúde pelos Residentes junto com e equipe da UBS tem conseguido fazer do grupo HiperDia um espaço pautado na Política Nacional de Promoção da Saúde, visando um atendimento multiprofissional e integrado.

Acrescenta-se a isto, a participação da pessoa idosa, no que tange à sua saúde e ao controle/acompanhamento de doenças crônicas, contribuindo efetivamente com a autonomia e a independência, com vistas à efetivação do envelhecimento ativo e com qualidade.

\section{REFERÊNCIAS}

1. Assis M. Envelhecimento ativo e promoção da saúde: reflexão para as ações educativas com idosos. Revista APS [Internet]. 2005 [citado em 30 ago 2016]; 8(1):15-24. Disponível em: http://www.ufjf.br/nates/files/2009/12/En velhecimento.pdf.

2. Ministério da Saúde (Br). Secretaria Nacional de Assistência à Saúde. ABC do SUS: doutrina e princípios [Internet]. Brasília, DF: Ministério da Saúde; 1990 [citado em 01 set 2016]. Disponível em: http://www.pbh.gov.br/smsa/bibliografia/a bc_do_sus_doutrinas_e_principios.pdf.

3. Ministério da Saúde (Br). Política Nacional de Atenção Básica [Internet]. Brasília: Ministério da Saúde; 2012. [citado em 30 ago 2016]. (Série E: Legislação em Saúde). Disponível

em:

http://189.28.128.100/dab/docs/publicacoe s/geral/pnab.pdf.

4. Ministério da Saúde (Br). Secretaria de Assistência à Saúde. Coordenação de Saúde da Comunidade. Saúde da Família: uma estratégia para a reorientação do modelo assistencial. Brasília: Ministério da Saúde; 1997.

5. Ministério da Saúde (Br). Secretaria de Atenção à Saúde. Departamento de Atenção Básica. HiperDia: Sistema de Cadastramento e Acompanhamento de Hipertensos e Diabéticos: manual de operação: Versão $1.5 \mathrm{M}$ 02 [Internet]. Brasília: Ministério da Saúde; 2002 [citado em 01 set 2016]. Disponível em: http://saude.maringa.pr.gov.br/downloads/h iperdia/manualhierdia2.pdf.

6. Instituto Brasileiro de Geografia e Estatística. Pesquisa Nacional de Saúde 2013 [Internet]. Rio de Janeiro: IBGE; 2014 [citado em 09 nov 2016]. Disponível em: ftp://ftp.ibge.gov.br/PNS/2013/pns2013.pdf. 7.World Health Organization. Envelhecimento ativo: uma política de saúde. Gontijo $S$, tradutora. Brasília: Organização PanAmericana da Saúde; 2005.

8. Ministério da Educação (Br). Educação para saúde [Internet]. [citado em 30 ago 2016]. 
Disponível

em:

http://portal.mec.gov.br/seb/arquivos/pdf/li vro092.pdf.

9. Freire P. Pedagogia do oprimido. Rio de Janeiro: Paz e Terra; 1975.

10. Presidência da República (Brasil). Lei $\mathrm{n}^{\mathrm{o}}$ 8.842, de 4 de janeiro de 1994. Dispõe sobre a política nacional do idoso, cria o Conselho Nacional do Idoso e dá outras providências. D.O.U., Brasília, DF, 05 jan 1994 [citado em 01 set 2016]. Disponível em: http://www.planalto.gov.br/ccivil_03/leis/L8 842.htm.

11. Ferrari MAC. O envelhecer no Brasil. Mundo Saúde 1999; 23(4):197-202.

12. Ministério da Saúde (Br). Organização Pan-Americana da Saúde. Avaliação do plano de reorganização da atenção à hipertensão arterial e ao diabetes mellitus no Brasil. Brasília: Ministério da Saúde; 2004.

13. Souza BS, Chagas MS, Silva ES, Domingos CB. Grupo de HiperDia: educando para vida.
Rev Pesqui Cuid Fundam. (online). out/dez 2010 [citado em 09 nov 2016]; 2(Supl):401$404 . \quad$ Disponível em: http://www.seer.unirio.br/index.php/cuidad ofundamental/article/view/959/pdf_162.

14. Buss P. Conceito de promoção da saúde e os determinantes sociais. Rio de Janeiro: Instituto de Tecnologia em Imunobiológicos; 2010 [citado em 30 ago 2016]. Disponível em: https://www.bio.fiocruz.br/index.php/artigo s/334-o-conceito-de-promocao-da-saude-eos-determinantes-sociais.

CONTRIBUIÇõES
Priscila Maitara Avelino Ribeiro realizou
levantamento bibliográfico e redação do artigo.
Mariana Campos de Sousa atuou na revisão
bibliográfica e redação do artigo. Marta Regina
Farinelli responsabilizou-se pela redação e
revisão crítica do artigo.

\section{Como citar este artigo (Vancouver)}

Ribeiro PMA, Sousa MCS, Farinelli MR. Envelhecer com qualidade e participação: experiência de um curso de residência multiprofissional em saúde. REFACS [Internet]. 2017 [citado em inserir dia, mês e ano de acesso]; 5(Supl. 2):311-317. Disponível em: inserir link de acesso. DOI:

\section{Como citar este artigo (ABNT)}

RIBEIRO, P. M. A.; SOUSA, M. C. S.; FARINELLI, M. R. Envelhecer com qualidade e participação: experiência de um curso de residência multiprofissional em saúde. REFACS, Uberaba, MG, v. 5, Supl. 2, p. 311-317, 2017. Disponível em: <link de acesso>. Acesso em: inserir dia, mês e ano de acesso. DOI:

\section{Como citar este artigo (APA)}

Ribeiro, P.M.A., Sousa, M.C.S. \& Farinelli, M.R. (2017). Envelhecer com qualidade e participação: experiência de um curso de residência multiprofissional em saúde. REFACS, 5(Supl. 2), 311-317. Recuperado em: inserir dia, mês e ano de acesso de inserir link de acesso e DOI. 